\title{
Sobre el sujeto-víctima: configuraciones de una ciudadanía limitada*
}

\author{
Diana Fuentes-Becerra* \\ Clara Atehortúa-Arredondo"*
}

Recibido: agosto de 2015

Evaluado: septiembre de 2015

Aprobado: noviembre de 2015

\section{RESUMEN}

Una de las virtudes que se le han atribuido a la justicia transicional en los países donde se ha implementado es su capacidad de fortalecer las instituciones y contribuir con el afianzamiento de la democracia. Una de las maneras de potenciar tanto la democracia como el restablecimiento de las víctimas ha sido propiciar su participación dentro de los procesos de justicia transicional y otras políticas públicas cuya finalidad es propender por las garantías de sus derechos. No obstante, la participación democrática de las personas está mediada por la calidad que se les otorga a los sujetos que participan. El objeto de este texto es proponer la necesidad de tener un debate acerca de las implicaciones que trae la forma en que se concibe a las "víctimas" en su participación dentro de procesos de reparación. Este documento concluye poniendo en consideración algunos puntos que podrían hacer parte del debate sobre la conceptualización de víctima y su participación.

Palabras clave: concepto de víctima; participación ciudadana; justicia transicional.

Este artículo es producto de las investigaciones de las tesis doctorales de las autoras iniciadas en el mes de febrero de 2011, las que se encuentran en desarrollo actualmente. "La categoría de víctima del conflicto armado colombiano: configuraciones globales, adaptaciones nacionales y realidades locales" y "La suspensión de los derechos de la población en situación de desplazamiento: ¿̇la única posibilidad para su restablecimiento?

** Abogada de la Pontificia Universidad Javeriana. Magíster en Derecho de la Universidad de Los Andes. Candidata a doctora del Doctorado en Derecho de la Universidad del Rosario. fuentesb.diana@urosario.edu.co

** Abogada de la Universidad de Medellín. Magíster en Ciencia Política de la Universidad de Antioquia. Candidata a doctora del Doctorado en Derecho de la Universidad del Rosario. Profesora de la Facultad de Derecho y Ciencias Políticas de la Universidad de Antioquia. clara.atehortua@udea.edu.co 


\title{
About the Subject-Victim: Configuration of a Limited Citizenship
}

\begin{abstract}
One of the virtues conferred upon the transitional justice in the countries where it has been implemented is its capacity to strengthen institutions and democracy. One of the ways to strengthen democracy and indemnify victims is to encourage their participation in transitional justice processes and other public policies intended to assure their rights. Democratic participation of people, however, is mediated by the quality granted to participating subjects. The objective of this article is to propose the need for having a debate on the consequences brought by the way "victims" are conceived during their participation within reparation processes. Finally, this document puts into consideration some points that could be part of the debate on the conceptualization of victims and their participation.
\end{abstract}

Key words: Concept of victim; citizen participation; transitional justice. 


\section{INTRODUCCIÓN}

Una de las virtudes que se le ha atribuido a la justicia transicional en los países donde se ha implementado es su capacidad de fortalecer las instituciones y contribuir con el afianzamiento de la democracia (De Greiff, 2011). Dicha contribución se hace a través de apoyar elementos que se consideran necesarios en la democracia, uno de los cuales es el reconocimiento de las víctimas como personas que sufrieron vulneraciones (De Greiff, 2011) y que, conforme a ellas, deben ser reparadas y restauradas en sus derechos. Las vulneraciones de derechos que sufren las víctimas se han definido como ataques directos contra su ciudadanía, siendo este un punto central para lograr la reparación de las víctimas. De ahí que uno de los objetivos de la justicia transicional sea fortalecer el restablecimiento de la ciudadanía de las víctimas.

Una de las maneras de potenciar tanto la democracia como el restablecimiento de las víctimas ha sido propiciar su participación dentro de los procesos de justicia transicional y otras políticas públicas cuya finalidad es propender por las garantías de sus derechos. A través de vincular a los beneficiarios a estos procesos, se pretende que la voz de las víctimas quede plasmada dentro de los proyectos que buscan su reparación, así como fortalecer sus capacidades en términos de su participación política. De esta manera las políticas públicas cuentan con una mayor legitimidad y los participantes de las discusiones y decisiones tomadas dentro de estos procesos tienen un escenario en el cual poner en juego sus capacidades ciudadanas.

No obstante, la participación democrática de las personas está mediada por la calidad que se les otorga a los sujetos que participan. La posibilidad de deliberar y de decidir está reservada para quienes tienen la calidad de ciudadanos plenos, es decir, personas con información y capacidad suficiente para tomar las decisiones pertinentes en los temas que tienen que ver con la autodeterminación de sus vidas como individuos y como parte de un colectivo (Holston E Appadurai, 1996). La determinación de quienes cuentan con estas características está dada por cada ordenamiento jurídico en la Constitución y la ley. Si bien quienes no cuentan con la totalidad de los requisitos tienen posibilidades de participar en procesos de carácter democrático, no pueden hacerlo de la misma forma de aquellos que cuentan con la calificación de ciudadanos plenos. Las víctimas llegan a los procesos de restablecimiento de derechos bajo el supuesto de que su ciudadanía ha sufrido menguas. Esta concepción tiene repercusiones en la forma en que se involucran las personas dentro de las políticas de reparación, específicamente en la manera en que participan dentro de las mismas.

Hasta el momento, los cuestionamientos sobre la participación de las víctimas se han centrado en las limitaciones de los mecanismos para incluir a la población víctima dentro de las discusiones y decisiones en los temas que les atañen (Berrío, 2013; Granada Vahos E González Díaz, 2009; Lemaitre, 2013; Naranjo-Gi- 
raldo, Morales-Lopera \& Granada-Vahos, 2009). Sin embargo, el papel que juega la concepción de "víctima" dentro de los procesos de participación ha sido mencionado solo tangencialmente. El objeto de este texto es proponer la necesidad de tener un debate acerca de las implicaciones que trae la forma en que se concibe a las "víctimas" en su participación dentro de procesos de reparación. Con esta finalidad, se harán algunas consideraciones en torno al sujeto víctima y cómo estas pueden condicionar su participación en las políticas y, por tanto, afectar la reparación integral de los derechos de los beneficiarios de las políticas. El texto tiene tres partes: la primera plantea una corta revisión sobre la manera en que se ha estudiado la participación de las víctimas; la segunda se propone el contexto de justicia transicional como adecuado para este debate, y la tercera, pone en consideración algunos puntos que podrían hacer parte del debate sobre la conceptualización de víctima y su participación en Colombia.

\section{SOBRE LA PARTICIPACIÓN DE LAS VÍCTIMAS. PUNTO DE PARTIDA}

Diversos escritos relacionan la categoría de víctima con la pérdida de la ciudadanía (Agamben, 2004; Holston \& Appadurai, 1996; Holzer, 2013). Las personas en situación de víctimas tienen un cambio en cuanto al ejercicio de sus derechos, que se denomina en términos de ciudadanías precarias, incompletas o en el bajo acceso a los derechos. En aras de evitar una mayor vulneración de la ciudadanía y en razón de la exigencia de ampliación de la democracia para los gobiernos de los países que tienen víctimas por vulneración de los derechos humanos e infracciones al derecho internacional humanitario, uno de los asuntos en los cuales se pone mayor atención es en la forma en que participan las víctimas de la implementación de las políticas con las que se espera lograr la reparación y la no repetición.

En Colombia desde la promulgación de la Ley 387 de 1997 , la cual marca el comienzo de la implementación de la política pública para la atención al desplazamiento forzado, hasta lo más reciente en relación con la Ley 1448 de 2011, la participación de los beneficiarios en la elaboración e implementación de las políticas ha sido un punto central en los análisis de políticas públicas (Berrío, 2013). Con el objeto de examinar la eficacia de la participación de los beneficiarios, las evaluaciones han tenido en cuenta diversos aspectos sobre la manera en que se vincula a los beneficiarios en el ciclo de la política pública, entre ellos: los momentos en que son llamados para ser vinculados a la discusión en relación con los temas de las políticas, el tipo de participación que se les otorga a las víctimas por los entes encargados de vincularlos, los instrumentos utilizados para la convocatoria y la participación de las personas, el carácter deliberativo o decisorio de la participación. Estos son algunos de los aspectos que se tienen en cuenta para establecer la manera en que participan las víctimas en las políticas para su restablecimiento. Los análisis afirman que la participación de las víctimas es baja y que la posibilidad 
de fortalecer la democracia no es eficaz, con base en el examen de los mecanismos de participación (Berrío, 2013; GranadaVahos \& González-Díaz, 2009).

En relación con la calidad de víctima, las evaluaciones de política pública toman como punto de partida la forma en que la normativa consagra quién es víctima. Dicha consagración se ha visto como un límite para la participación de quienes deben estar vinculados con las políticas públicas; muchas de las víctimas, por diversas razones, no están incluidas dentro de los registros establecidos y no pueden participar. De esta manera, gran parte de la población que debe estar vinculada a las políticas públicas queda excluida previamente. Esta situación implica una reducción significativa del universo de personas que debe participar dentro del ciclo de las políticas para que se pueda hablar de un afianzamiento de la democracia y apoyar el mejoramiento de las capacidades ciudadanas de las víctimas. Si bien los requisitos que establece el ordenamiento jurídico para determinar los beneficiarios de la reparación son un límite para la participación, debido a que este criterio excluye a quienes no cumplen con los parámetros legales, este texto no pretende cuestionar la forma en que se ha tipificado a las víctimas para ser incluidas en la política, sino las consecuencias que tiene para la participación el hecho de ser denominado víctima. Con esta finalidad, se explicará por qué es necesario en el actual contexto de justicia transicional plantear la discusión en torno a quién es víctima. Luego, se harán algunas consideraciones respecto a los conceptos de víctima y la forma en que estas concepciones pueden condicionar la participación de los beneficiarios en la política pública. Por último, se explicará la forma en que estas discusiones pueden afectar la reparación integral de los derechos de las víctimas.

\section{LUGAR DE LA JUSTICIA TRANSICIONAL COMO ESPACIO PARA LA NORMALIZACIÓN DE LA JUSTICIA. PUNTO DE DEFINICIÓN}

Actualmente, la justicia se desenvuelve en contextos que rompen con los estándares para que funcione en condiciones de normalidad. La normalidad se fundamenta en la existencia de acuerdos en torno a tres puntos básicos: (i) qué se puede reclamar a través de ella; (ii) quién puede presentarse ante ella y (iii) los mecanismos a los cuales se debe acceder (Fraser, 2008, pág. 99). La posibilidad de llegar a acuerdos globales sobre estos asuntos es cada vez más extraña. La globalización, los cambios en el orden internacional político, económico y social, el cuestionamiento en relación con el establecimiento y funcionalidad de las fronteras, las guerras entre países y los conflictos internos, entre otros, hacen difícil tener "una visión compartida" (Fraser, 2008, pág. 105) en relación con los asuntos que afectan el funcionamiento de la justicia en términos normales. La inexistencia de acuerdos permite la ampliación de puntos que podrían extender los límites de acceso a la justicia para grupos que han sido excluidos, a través de su inclusión y de la modificación de los instrumentos para su ingreso en términos de equidad. De esta manera, se pueden redefinir los límites 
de los asuntos que se pretende discutir, las personas que pueden acudir a esta discusión y los mecanismos adecuados para hacerlo.

En Colombia, no puede hablarse de un contexto de justicia normal desde hace más de 50 años. Esta imposibilidad no solo proviene de un contexto perturbado en el que lo establecido en el ordenamiento jurídico no se ha aplicado, sino que los hechos que han servido de excusa para esa inaplicación dan cuenta del desacuerdo sobre las premisas que son presupuestos para la justicia normal. Tal es el caso de la dinámica del conflicto armado, de los continuos estados de sitio y de la forma en que se le ha dado tratamiento a los conflictos políticos y sociales.

La existencia de estas circunstancias obstaculiza el establecimiento de la justicia formal, y le ha dado prevalencia al aspecto negativo de la anormalidad de la justicia: la imposibilidad de tener un marco estable para la solución de desacuerdos entre los miembros de la sociedad (Fraser, 2008). El miedo a la eliminación física o política, la ineficacia de los mecanismos de deliberación y la suspensión de las garantías para las libertades de expresión han hecho que no se pueda establecer una discusión en torno a las premisas que son presupuestos para el ejercicio de una justicia en términos normales. Estas discusiones se han postergado indefinidamente, sin que se haya dado la oportunidad para discutir ampliamente estos asuntos.

Si bien no existe una variación notable sobre estas circunstancias, en este mo- mento se abre la posibilidad de entablar una discusión en relación con estos aspectos. Esta viabilidad está dada por el momento político creado a partir de la instauración -al menos en términos formales- de la justicia transicional a través de la Ley 1448 de 2011. Más allá de las críticas a la implementación de este tipo de mecanismos en otros contextos, tales como el de Suráfrica en donde los procesos de verdad dieron lugar a que a través del lenguaje la situación de víctima se constituyera en un parte de la identidad de las personas (Segall, 2002), la instauración de la justicia transicional abre consigo dos posibilidades: de una parte, les da viabilidad política a las discusiones y a la puesta en audiencias públicas de los diversos testimonios y las distintas posiciones que han sido silenciados por las circunstancias antes mencionadas (Segall, 2002); de la creación de este espacio se deriva la segunda posibilidad, esto es, abrir un debate en torno a las premisas de la justicia-el objeto de la reclamación, quién puede hacerlo y los medios para ello-. Así las cosas, aparece un espacio político favorable para que prevalezca el aspecto positivo de la justicia anormal. En este sentido, se cuenta con una oportunidad para avanzar en la definición conjunta de los aspectos clave para que esta sirva a la normalidad en el momento en que fácticamente sea posible. Para plantear este debate, es necesario tener en cuenta la forma en que se han conceptualizado las víctimas del conflicto armado. A continuación, se presentan algunas de las consideraciones frente a esa discusión. 


\section{LA CATEGORIA DE VÍCTIMA DE CONFLICTOS INTERNOS}

Según Mate la visibilidad de las víctimas corresponde a un "fenómeno epocal, un signo de nuestro tiempo" (Mate, 2008, p. 21). Ese cambio de época lo marcaría la devastación generada por los totalitarismos en la Segunda Guerra Mundial, hito que representó una nueva forma de entender la figura de la víctima. Así lo describe Hartog quien contrapone la víctima antigua a la víctima moderna. La víctima antigua se relaciona con el sacrificio y la noción de mártir, ya que en cierta medida se consideraba víctima a aquellos que morían ya sea por la ciudad-Estado o por la patria (Hartog, 2012). En contraposición a esta figura, se encuentra la víctima moderna, que tiene como principal referente el exterminio judío. Así, pues, se reemplaza la idea heroica de la víctima por la de la figura de "las víctimas innombrables, a las que compadecemos, las que han padecido, que no han podido más que padecer, que no han hecho sino padecer. En pocas palabras, hasta este momento activa y positiva, la noción de víctima se carga de una connotación pasiva" (Hartog, 2012, pág. 13). Esa pasividad está ligada a la primera característica destacada por Mate de la víctima: la inocencia. Para Mate el sufrimiento que experimenta un ser humano lo constituye en víctima solo cuando fue causado por el hombre, sin razón alguna (Mate, 2008, p. 35).

Las consecuencias de esta conceptualización de la víctima han sido analizadas por diversos autores, desde diferentes perspectivas. Por un lado, podemos encontrar a Orozco (2005) y Bouris (2007) quienes proponen una lectura más realista de los conflictos armados internos, en los cuales no se replica el modelo del Holocausto, y donde es posible encontrar procesos de victimización más complejos de aquellos donde hay una clara línea entre víctimas y victimarios. Asimismo, el trabajo de Bouris demuestra que la difusión por diferentes medios de imágenes correspondientes al estereotipo de las víctimas como inocentes, puras y superiores moralmente (por ejemplo con proclividad a perdonar y dispuestas a la reconciliación) termina en muchos casos excluyendo de protección a quienes no cumplen con tales criterios.

Por su parte, los trabajos de Wilson (1997) y Mutua (2001) plantean que los informes de derechos humanos, principal instrumento de denuncia de violaciones a los derechos humanos e infracciones al derecho internacional humanitario DIH-, reproducen representaciones de las víctimas como seres necesitados de protección, pasivos e inocentes. Este tipo de representaciones tiene un doble impacto: por un lado, estimula a las víctimas a presentarse en estos términos para efectos de acceder a la atención (Merry, 2007), y por otro, propicia que los defensores de las víctimas se constituyan en los voceros de "los que no tienen voz". Malkki analizó este tipo de relaciones durante su etnografía en un campo de refugiados hutus en Tanzania, donde encontró que los administradores del campo imaginaban a los refugiados como 
un tipo particular de persona: una víctima cuyo juicio o razón está comprometido por sus experiencias traumáticas. Esta caracterización generaba la necesidad de la intervención profesional y desplazaba la voz de la víctima (Malkki, 1996).

En una línea similar es posible reseñar a Fassin sobre el trabajo humanitario en el conflicto palestino-israelí. Este autor demuestra cómo la definición de la subjetividad política de las víctimas está mediada por una constante referencia al trauma, entendido no solo en su registro clínico, sino como un instrumento que permite la producción de testimonios sobre la base de la experiencia de víctimas de la violencia. La definición de la subjetividad política para Fassin se relaciona con el paradigma de Althusser de interpelación, debido a que la designación social de un colectivo constituye al mismo tiempo un llamado al sujeto a identificarse a sí mismo -en ocasiones en contra de su voluntad- con la forma en que es designado. De este modo, para Fassin la forma predominante de entender a la víctima desde el trabajo humanitario está ligada al trauma y con ello lo que se logra es contribuir a la formación de una subjetividad política que requiere de la voz de otro (de los trabajadores humanitarios) para expresarse. Así, según este autor "en el mundo contemporáneo la proliferación del humanitarismo incrementa de forma paralela el silencio del sobreviviente" (Fassin, 2008).

\section{REFLEXIONES SOBRE LAS FORMAS EN QUE SE HA CONFIGURADO LA CATEGORÍA DE VÍCTIMA EN EL CONTEXTO COLOMBIANO}

A pesar de la diferenciación que puede establecerse entre los contextos analizados por cada uno de los autores reseñados y el caso colombiano, es pertinente analizar cómo se ha ido llenando de contenido a la categoría de víctima del conflicto colombiano. Para contribuir con esta necesaria tarea presentamos a continuación algunas ideas que buscan provocar la discusión en relación con la forma predominante en que esta categoría viene siendo entendida.

\section{PROBLEMAS PARA EL RECONOCIMIENTO}

En medio de la discusión por la exclusión de la atención, las sentencias de constitucionalidad de la Ley 1448 de 2011 ampliaron el universo de las víctimas dentro del marco de la ley, en donde se mantiene que la calidad de víctima es una condición de hecho derivada del daño generado por las violaciones graves y manifiestas a las normas internacionales de derechos humanos o por las infracciones al derecho internacional humanitario. Asimismo, establece que la mera declaración de la persona es válida para ser considerada víctima y, en caso tal de que haya dudas sobre esta situación, el Estado debe desvirtuarlo a través de un proceso con contradicción, en donde la carga de la prueba está en cabeza de este. De esta manera, la discusión acerca de quién se traslada al escenario de la justicia, debe darse en los términos de la justicia transicional. 
Sin perder de vista que los conflictos sobre quién pertenece al universo de víctimas no han sido superados y que todos los días existen reclamaciones para lograr la inclusión dentro de los registros de víctimas, y atendiendo a que es uno de los temas que ha suscitado más acciones judiciales por parte de quienes se ven como excluidos de este universo, es necesario centrarse en un punto que se ha dejado de lado en medio de la discusión por el acceso a la atención: las consecuencias de la construcción del sujeto y de la recuperación de la ciudadanía. Esta polémica incluye la discusión en torno al reconocimiento y la representación de las víctimas (Fraser, 2008, pág. 112), y de ella depende la participación equitativa e imparcial de todos los que se ven afectados dentro del debate.

El primer punto que debe tenerse en cuenta es que la categoría de víctima tiene un carácter homogeneizador (Berrío, 2013). A partir de que las personas son reconocidas como víctimas, el hecho de haber sufrido la vulneración de sus derechos prima en la definición de quiénes son. De esta manera se iguala a las personas, desconociendo características tales como el sexo, la orientación sexual, la edad, la etnicidad, entre otros. El desconocimiento de estas circunstancias impide identificar la situación de vulnerabilidad en la que se encuentran las personas debido a los procesos de marginación social, económica y simbólica que han sufrido, derivados de poseer una o varias de estas características. Esta negación tiene consecuencias en diferentes ámbitos: en el de la reparación de derechos es un obstáculo a los procesos de memoria, pues el desconocimiento del papel que jugaron la vulnerabilidad y la marginación en los procesos de violencia contra grupos específicos de la población niega la posibilidad de exponer la verdad de los hechos victimizantes que sufrieron quienes pertenecían a estos grupos (Corporación Humanas, 2013); en los procesos de participación en la política pública de atención, el desconocimiento de las características que diferencian a las víctimas y su relación con el grado de marginación que han sufrido en otros procesos de participación no permite establecer mecanismos efectivos para que sean tenidas en cuenta en la toma de decisiones durante la aplicación de la política. Este hecho es uno de los motivos que ha puesto en tela de juicio la legitimidad de los procesos de participación que se llevan hasta el momento en el marco de la Ley 1448 de 2011 (Berrío, 2013).

Otro asunto a tener en cuenta es entender la victimización como una característica identitaria de la población. Desde esta perspectiva, el hecho de haber sufrido la vulneración se convierte en un rasgo constitutivo de quien está dentro de la categoría de víctima. En este sentido, lo que es una experiencia que debe ser superada se convierte en una condición de existencia. En consecuencia, el reconocimiento de la calidad de víctima se convierte en una contradicción con el objeto de la reparación integral.

Ligado con lo anterior, un tercer tema tiene que ver con la permanencia en la situación de la calidad de víctima para lograr la 
reparación. El objetivo de los programas de reparación de derechos en el contexto de la justicia transicional es la superación de la situación de víctima. No obstante, dentro de los diseños de los sistemas de atención a las víctimas, los mecanismos creados para la participación y la consecución de la verdad de lo sucedido y los procesos judiciales para la declaración de derechos son espacios donde se perpetúa la situación de víctimas. Durante el período en el que están dentro de estos procesos, lo que estas personas hacen es insistir en la condición de víctimas como esencial para estar allí; de esta manera, el reconocimiento se torna en una forma de existencia, y en prerrequisito para la interpelación dentro de estos espacios (Segall, 2002).

Como correlato al problema del reconocimiento está el de la representación. En términos generales se habla de una representación fallida cuando no es posible la participación equitativa de los miembros de un universo. En el caso de las víctimas, constantemente se refiere a una representación de este tipo. Esta falencia está dada por la existencia de diversos obstáculos, entre ellos, el funcionamiento de los mecanismos dispuestos para la participación de las víctimas. No obstante, la forma en que se dan el reconocimiento y el concepto de víctima pueden crear otro tipo de obstáculos. De la manera en que se conciben estos dos puntos, se otorga o se disminuye capacidad a las víctimas para la deliberación y la toma de decisiones dentro de los espacios de la justicia transicional. Una concepción de víctima que ponga a todos los beneficiarios como sujetos con una capacidad política incompleta traerá como consecuencia un reconocimiento fallido (Fraser, 2008).

El artículo 2 de la Ley 1448 de 2011 o Ley de Víctimas estipula: "la presente ley regula lo concerniente a ayuda humanitaria, atención, asistencia y reparación de las víctimas de que trata el artículo 3 de la presente ley, ofreciendo herramientas para que estas reivindiquen su dignidad y asuman su plena ciudadanía". La caracterización de las víctimas como sujetos incompletos que "debe[n] asumir su ciudadanía plena" inscribe a las víctimas en una condición no-política en el terreno de lo humanitario, terreno en el cual el daño causado en el marco del conflicto impide que se construya una voz propia $y$, por ende, se necesita de un tercero que hable por ellos, como se expuso anteriormente.

A partir de esta construcción se definen instancias de participación donde la posibilidad de decidir de esta población está restringida (Fraser, 2008), pues tal posibilidad se les entrega a otros sujetos que deciden por ellos. En los espacios de justicia transicional, ese sujeto está definido institucionalmente por los "tecnócratas de la justicia" (Fraser, 2008). A ellos se les entrega la decisión de las disputas sobre el quién, entendido como la definición de cómo se concibe al sujeto, a quién se incluye y de qué manera debe ser representado en los espacios de decisión. 


\section{CONCLUSIÓN: CONTINUIDADES Y DISCONTINUIDADES ENTRE EL SISTEMA DE ATENCIÓN A LA POBLACIÓN DESPLAZADA Y LA LEY DE VÍCTIMAS}

A pesar de que desde la Ley 418 de 1997 se consagró en el ordenamiento jurídico colombiano la categoría de víctima de hechos violentos que se susciten en el marco del conflicto armado interno, el eje respecto del cual se diseñó la mayoría de políticas públicas fue el desplazamiento interno. En este sentido, la adopción de la Ley 1448 de 2011 condujo a la creación de un conjunto de entidades y de protocolos encaminados a implementar los mandatos de esta ley, trayendo consigo el nuevo lenguaje de la justicia transicional que, si bien desde la Ley 975 de 2005 (Ley de Justicia y Paz) y el Decreto 1290 de 2008 (Decreto de la Presidencia de la República de Colombia para la Reparación Administrativa) se venía discutiendo, pasó a tener una preponderancia fundamental en las relaciones entre las víctimas y el Estado.

Las formas en que las víctimas han recibido este cambio ha sido objeto de análisis desde el punto de vista psicosocial en trabajos como el de Villa. Para este autor, que estudia los escenarios de reparación, verdad y justicia propiciados por el Estado colombiano hasta junio de 2012 en un marco donde el conflicto aún persiste, lo que puede afirmarse es que estos escenarios no han generado una resignificación de los hechos violentos experimentados $y$, por ende, se han propiciado en muchos casos procesos de revictimización. (Villa, 2013)
Sin perder de vista que este autor discute muchos puntos, quisiéramos rescatar un argumento que utiliza Villa en el texto analizado, y que constituye un punto de partida de muchas intervenciones con víctimas:

[...] existe una construcción precaria de ciudadanía de derechos por parte de buena cantidad de las víctimas de violencia política en Colombia, la mirada de sí mismos/as como 'receptores de ayuda por parte del Estado', especialmente en las bases sociales de los colectivos analizados, la búsqueda y recepción de este dinero (la reparación administrativa) no se logra analizar ni se logra reflexionar sobre los términos de la relación e interlocución con el Estado. No se logra comprender como una lucha y una reivindicación por los derechos, sino que se recibe como una 'ayuda', a la manera de un subsidio, con la que no se contaba, ante la cual se deben hacer los trámites pertinentes, pero que no repara en realidad ni implica una acción de justicia restaurativa ni distributiva por parte del Estado, se recibe más como una 'caridad' (Villa, 2013, pág. 313).

El reto que plantea este argumento es la necesidad de tener en cuenta los contextos en que buscan ser aplicados los "paquetes" de la justicia transicional. Así las cosas, un aspecto que merece ser tenido en cuenta está constituido por la transformación que supone la política de atención a la población desplazada que se ejecutó por más de 15 años. Durante este período, es decir, desde que se institucionalizaron las medidas de atención a las personas en situación de desplazamiento en el año de 1997, gracias a un complejo sistema configurado por leyes, decretos y 
órdenes jurisprudenciales, se manejaron tres fases de atención que buscaban la superación del estado de vulnerabilidad de las víctimas. El uso recurrente de términos como la "ayuda humanitaria" y sus "prórrogas" era -y es- un lugar común en la cotidianidad de las personas afectadas por el conflicto. Sumado a lo anterior, la promesa de la "auto-sostenibilidad" encarnada en una serie de cursos encaminados a obtener el "capital semilla" para proyectos productivos también es una realidad que ha sido parte durante mucho tiempo de lo que significaba ser víctima en nuestro país. De ahí que en nuestro criterio se debe, en un primer momento, proponer lecturas que permitan entender las posiciones concretas de víctimas específicas en relación con el Estado y con entidades no gubernamentales a partir de una mirada compleja que dé cuenta de las contradicciones, prejuicios y estereotipos que definen la interpelación a las víctimas, así como de las formas en que estas responden a este llamado.

\section{BIBLIOGRAFÍA}

Agamben, G. (2004). Estado de excepción. Homo Sacer II, 1. Madrid: PRE-TEXTOS.

Berrío, J. M. (2013). Público. Las mesas de participación de víctimas : ¿ Una frustración más o un mecanismo de. Revista de Derecho Publico, (Número 31), pp. 2-33.

Bouris, E. (2007). Complex Political Victims. Bloomfield: Kumarian Press.

Corporación Humanas. (2013). La violencia sexual. Una estrategia paramilitar. Bogotá: Corporación Humanas.
De Greiff, P. (2011). Algunas reflexiones acerca del desarrollo de la Justicia Transicional. Anuario de Derechos Humanos, (Número 2), pp. 17-39. Retrieved from www.anuariocdh.uchi.cl

Fassin, D. (2008). The humanitarian politics of testimony: Subjectification through Trauma in the Israeli-Palestinian Conflict. Cultural Anthropology, Volumen 23, (Número 3), pp. 531-558

Fraser, N. (2008). Escalas de la justicia. Barcelona: Herder.

Granada-Vahos, J., E Gonzalez-Diaz, S. (2009). Acción colectiva de las organizaciones de población desplazada en Medellín. Estudios Políticos, (Número 35), pp. 107-130.

Hartog, F. (2012). El tiempo de las víctimas. Revista de Estudio Sociales, (Número 44), pp. 12-19.

Holston, J., E Appadurai, a. (1996). Cities and Citizenship. Public Culture, Volumen 8, (Número 2), pp. 187-204. http://doi. org/10.1215/08992363-8-2-187

Holzer, E. (2013). What Happens to Law in a Refugee Camp? Law \& Society Review, Volumen 47, (Número 4), pp. 837-872. http://doi. org/10.1111/lasr.12041

Lemaitre Ripoll, J. (2013). Diálogo sin debate: la participación en los decretos de la ley de víctimas. Revista de Derecho Público, (Número 31), pp. 3-36.

Malkki, L. H. (1996). Speechless Emissaries: Refugees, Humanitarianism, and Dehistoricization. Cultural Anthropology, Volumen 11, (Número 3), pp. 377-404.

Mate, R. (2008). Justicia de las víctimas: terrorismo, memoria, reconciliación. Barcelona: Anhtropos Editorial.

Merry, S. E. (2007). Introduction: Conditions of vulnerability. En M. Goodale, \& S. E. Merry (Edits.), The practice of human rights: Tracking Law 
between the Global and the Local (págs. 195-203). Cambridge: Cambridge University Press.

Mutua, M. (2001). Savages, Victims and Saviors: the metaphor of human rights. Harvard International Law Journal, Volumen 42, (Número 1), pp. 201-245.

Naranjo-Giraldo, G., Morales-Lopera, J. E., E Granada-Vahos, J. (2009). Las políticas públicas territoriales como redes de política pública y gobernanza local : la experiencia de diseño y formulación de las políticas públicas sobre desplazamiento forzado en el departamento de Antioquia y la ciudad de Medellín. Estudios Políticos, (Número 35), pp. 81-105.

Orozco, I. (2005). Sobre los límites de la conciencia humanitaria: dilemas de la paz y la justicia en América Latina. Bogotá: Temis, Uniandes.
Sanchez Medina, L. A., \& Atehortua-Arredondo, C. (2008). Narraciones sobre la experiencia del éxodo. El caso del desplazamiento forzado en la comuna 13. Vniversitas, 117 (juliodiciembre de 2008), pp. 15-39.

Segall, K. W. (2002). Postcolonial performatives of Victimization. Public Culture, Volumen 14, (Número 3), pp. 617-619

Villa, J. D. (2013). Consecuencias psicosociales de la participación en escenarios de justicia transicional en un contexto de conflicto, impunidad y no-transición. Revista Ágora, Volumen 13, (Número 2), pp. 307-338.

Wilson, R. (1997). Representing human rights violations: social contexts and subjectivities. En R. A. Wilson (Ed.), Human Rights, Culture and Context. Anthropological Perspectives (págs. 134-160). London: Pluto Press. 
